\title{
Volume of fluid model applied to curved open channel flows
}

\author{
T. Patel \& L. Gill \\ Department of Civil, Structural and Environmental Engineering, \\ Trinity College, University of Dublin, Ireland.
}

\begin{abstract}
An attempt has been made to simulate secondary flows in curved open channels using three-dimensional CFD analysis. Besides the classical center-region cell, a counter rotating outer bank cell is often observed which could play an important role in the mechanism of sediment transport. The CFD analysis is carried out on the $120^{\circ}$ curved open channel bend using the commercial software package Fluent. The volume of fluid (VOF) model is used to simulate the air-water interaction at the free surface and the turbulence closure was obtained using the Reynolds stress model (RSM). It is observed that the RSM model was able to predict both circulation cells successfully. The results show that the core of maximum velocities is found close to the separation between both circulation cells and below the free surface which agrees well with the experimental data.

Keywords: open channel, secondary flows, computational fluid dynamics, volume of fluid, free surface, turbulence modeling.
\end{abstract}

\section{Introduction}

Secondary flows are a significant feature of flow in open-channel bends. They are formed due to a local imbalance between the pressure gradient and centrifugal force at any given section. They tend to redistribute the mean velocity, alter the boundary shear stress and erode the outer bank. Most of the research on flows in bends over the past decade has concentrated on the central portion of the flow, whereas the flow characteristic at the outer bank (where a small counter-rotating circulation cell is present) has often been neglected. Earlier studies indicate that the center-region circulation cell has been captured successfully using a two-equation turbulence model. Patel and Gill [1], 
Rameshwaran and Naden [2, 3] and Ye and McCorquodale [4] were able to simulate the same using the $\kappa-\varepsilon$ turbulence model. Booij [5] also concluded that $\kappa-\varepsilon$ turbulence model was unable to predict the outer bank cell but was able to reproduce the center-region cell. Blanckaert and Graf [6] stressed the importance of high-resolution experimentation to fully understand the mechanism underlying the generation of the outer bank cell and also mentioned that the lack of proper experimental data has hampered the verification of investigations to date by means of numerical investigation. Blanckaert and de Vriend [7] also found that the decreasing velocities towards the water surface $(\partial \mathrm{v} / \partial \mathrm{z}<0)$ and turbulent anisotropy are an important generation mechanism for the outer-bank cell. Unlike the two-equation model, the RSM model is capable of simulating cross-stream turbulent anisotropy in compound straight channels [8].

It has been also cited that the discretization schemes (for the non-linear terms in the momentum equations) can also be responsible for accurate results [9]. They indicated that the single order unwinding scheme might result in numerical diffusion especially if the flow is skewed relative to the numerical mesh. Nicholas [10] attempted to study the flow behaviour in straight open channels using Fluent [11] and also mentioned the importance of the grid resolution near the bottom wall to capture the detailed flow characteristics.

This paper reports the simulation of both circulation cells in a $120^{\circ}$ curved open channel using the RSM turbulence model. The air-water interaction is modeled using the VOF method as developed by Hirt and Nicholls [12]. It should also be noted here that VOF model has not been applied previously to the study of curved open channel flows.

\section{Problem formulation}

CFD analysis was carried out on the $120^{\circ}$ curved open channel experiment conducted by Blanckaert and Graf [6]. Figure 1 shows the geometrical layout and the cross-sectional details at the test section. The hydraulic parameters for the fluid flow are also shown in Table 1. The test case was selected to imitate the real flow conditions which include variable bed topography, as found in nature. The test location is at $60^{\circ}$ into the bend and the measurements are taken at the outer half of the section.

\subsection{Computational method}

The computations were performed on an adaptive grid using Fluent, a general purpose CFD software. The governing flow equations for mass and momentum conservations are as follows:

$$
\begin{gathered}
\frac{\partial \rho}{\partial t}+\frac{\partial}{\partial x_{i}}\left(\rho u_{i}\right)=0 \\
\frac{\partial\left(\rho u_{i}\right)}{\partial t}+\frac{\partial}{\partial x_{j}}\left(\rho u_{i} u_{j}\right)=-\frac{\partial p}{\partial x_{i}}+\frac{\partial}{\partial x_{j}}\left[\mu\left(\frac{\partial u_{i}}{\partial x_{j}}+\frac{\partial u_{j}}{\partial x_{i}}-\frac{2}{3} \delta_{i j} \frac{\partial u_{k}}{\partial x_{k}}\right)\right]+\frac{\partial}{\partial x_{j}}\left(-\rho \overline{u_{i}^{\prime} u_{j}^{\prime}}\right)_{i}
\end{gathered}
$$


Table 1: $\quad$ Experimental characteristics.

\begin{tabular}{|c|c|c|c|c|c|}
\hline $\begin{array}{c}\text { Radius of } \\
\text { Curvature } \\
(\mathrm{m})\end{array}$ & $\begin{array}{c}\text { Depth of } \\
\text { flow (m) }\end{array}$ & $\begin{array}{c}\text { Channel } \\
\text { width }(\mathrm{m})\end{array}$ & $\begin{array}{c}\text { Mean } \\
\text { Velocity } \\
\mathrm{U}(\mathrm{m} / \mathrm{s})\end{array}$ & $\begin{array}{c}\text { Discharg } \\
\text { e Q(1/s) }\end{array}$ & $\begin{array}{c}\text { Reynolds } \\
\text { Number }\end{array}$ \\
\hline 2 & 0.114 & 0.4 & 0.38 & 17 & 67260 \\
\hline
\end{tabular}

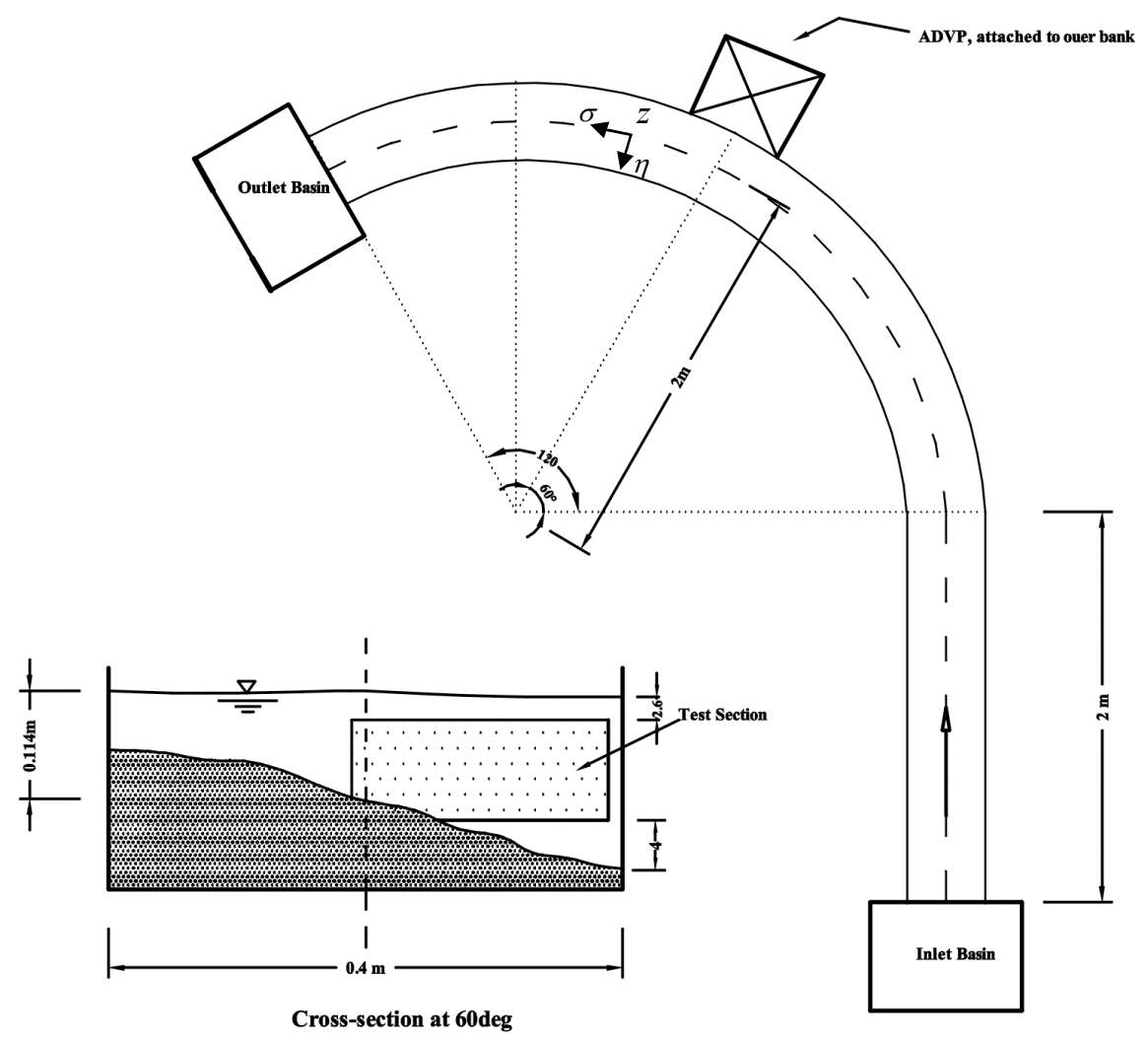

Figure 1: Geometric layout of channel. 
where, $\delta_{i j}$ is the Kronecker delta, and $-\rho \overline{u_{i}^{\prime} u_{j}^{\prime}}$ is the Reynolds stress. The Reynolds stress equation is given by:

$$
\begin{aligned}
& \underbrace{\frac{\partial}{\partial t}\left(\rho \overline{u_{i}^{\prime} u_{j}^{\prime}}\right)}_{\text {LocalTimeDerivative }}+\underbrace{\frac{\partial}{\partial x_{k}}\left(\rho u_{k} \overline{u_{i}^{\prime} u_{j}^{i}}\right)}_{C_{i j} \equiv \text { Convection }}=-\underbrace{\frac{\partial}{\partial x_{k}}\left[\rho \overline{u_{i}^{\prime} u_{j}^{\prime} u_{k}^{\prime}}+\overline{p\left(\delta_{k j} u_{i}^{\prime}+\delta_{i k} u_{j}^{i}\right)}\right]}_{D_{T, i j} \equiv \text { TurbulentDiffusion }} \\
& +\underbrace{\frac{\partial}{\partial x_{k}}\left[\mu \frac{\partial}{\partial x_{k}}\left(\overline{u_{i}^{\prime} u_{j}^{\prime}}\right)\right]}_{D_{L, i j} \equiv \text { MolecularDiffusion }}-\underbrace{\rho\left(\overline{u_{i}^{\prime} u_{k}^{i}} \frac{\partial u_{j}}{\partial x_{k}}+\overline{u_{j}^{\prime} u_{k}^{\prime}} \frac{\partial u_{i}}{\partial x_{k}}\right)}_{P_{i j} \equiv \text { Stress } \operatorname{Pr} \text { oduction }}+\underbrace{p \overline{\left(\frac{\partial u_{i}^{\prime}}{\partial x_{j}}+\frac{\partial u_{j}^{\prime}}{\partial x_{i}}\right)}}_{\phi_{i j} \equiv \text { Pr essureStrain }}-\underbrace{2 \mu \overline{\frac{\partial u_{i}^{\prime}}{\partial x_{k}} \frac{\partial u_{j}^{\prime}}{\partial x_{k}}}}_{\varepsilon_{i j} \equiv \text { Dissipation }}
\end{aligned}
$$

It should be noted here that the unsteady solver is used only to get the steady flow results, and not intended to obtain time-accurate solutions. Time derivative terms are discretized using the first order accurate backward implicit scheme. Convection terms are discretized using the third order Monotone upstreamcentered schemes for conservation laws (MUSCL) scheme, while diffusion terms are discretized using the second order accurate central differencing scheme. The pressure-velocity coupling is achieved using the SIMPLE algorithm [13].

As mentioned earlier, the VOF method has been employed to simulate the airwater interaction at the free surface. The VOF method which was developed by Hirt and Nichols [12] is a type of interface-capturing method which relies on the fact that two or more fluids/phases are not interpenetrating and for each additional phase, a new variable that is the volume fraction of the phase in the computational cell is introduced. The mass conservation equation for the $q^{\text {th }}$ phase is given by:

$$
\frac{\partial \alpha_{q}}{\partial t}+\vec{v} \cdot \nabla \alpha_{q}=0
$$

It should be noted here that the volume fraction equation is not solved for the primary phase, but is based on the constraint that in each cell, the volume fraction of all phases must sum to unity,

$$
\left(\sum_{q=1}^{n} \alpha_{q}=1\right) .
$$

A single momentum equation is solved throughout the domain, and the resulting velocity field is shared among the phases. The momentum equation depends on the volume fractions of all the phases through the fluid properties, which are determined by the presence of the component phases in each control volume, e.g.,

$$
\begin{gathered}
\rho=\alpha_{q} \rho_{q}+\left(1-\alpha_{q}\right) \rho_{p} \\
\mu=\alpha_{q} \mu_{q}+\left(1-\alpha_{q}\right) \mu_{p}
\end{gathered}
$$

where subscripts $p$ (air) and $q$ (water) denote the primary and secondary phases respectively for open channel flows. In addition, the complexity of implementing 
boundary conditions on the surface has been avoided since the water surface is the interface between the air and the water. For an unsteady fluid flow, the cells currently filled with air provide the space for the water when the water level rises. Thus, this method allows free movement of the water at the interface.

\subsection{Grid generation and boundary conditions}

Numerical grids were constructed with the Gambit preprocessor available in the CFD package Fluent. The flow domain was divided into number of nonoverlapping unstructured (T-grid) meshes. The total number of meshes increased to $1,144,662$ after grid adaptation. The boundary conditions for the flow domain are as follows:

I. Inlet. A new boundary condition 'Open Channel' (available in Fluent [11]) is defined at the inlet. Two separate inlets are defined for air and water with the same group ID. Mass flow rates are defined for both the phase depending upon the velocity and the inlet area. The depth of the flow is known in advance from the experimental results which help in defining the free surface level before starting the simulations. The flow domain is initialized with the volume fraction of secondary phase (i.e. water) equal to 1 up to the free surface level. This procedure also helps in the convergence of the problem.

II. Outlet. Pressure Outlet boundary condition is applied at the outlet. The pressure is kept at atmospheric pressure (i.e. gauge pressure $=0$ ). Here also two separate outlets are kept with the same group id.

III. Top Surface. As discussed earlier the boundary condition at the interface is avoided by using the VOF model. The top surface above which is air is initially kept at symmetry (a boundary condition) in which all the normal gradients $(\partial / \partial z=0)$ and the normal components are zero. Once the solution stabilizes the top surface boundary condition is changed to ambient pressure conditions to represent the real flow conditions more accurately.

IV. Wall. The bottom and side surfaces are defined as Wall boundary condition. In this, study, the standard wall function has been employed, which may be expressed as follows:

$$
\begin{gathered}
\frac{u_{p} u^{*}}{\tau_{w} / \rho}=\frac{1}{\kappa} \ln \left(E \frac{\rho u^{*} y_{p}}{\mu}\right)-\Delta B \\
u^{*}=C_{\mu}^{1 / 4} \kappa^{1 / 2} \\
\Delta B=\frac{1}{\kappa} \ln f_{r}
\end{gathered}
$$

where, $f_{r}$ is the measure of the roughness of the bed/wall. It should also be noted here that fine grid resolution near the wall has been avoided by using the wall function which also helps in reducing the computational time. 


\section{Results and discussion}

\subsection{Mean velocity field}

The comparisons between the experimental and the predicted flow fields are shown in Figure 2(a-d). As described earlier the test section is located at $60^{\circ}$ downstream into the bend and focused at the outer half of that section. The figure shows that both the circulation cells are captured successfully using the RSM model. Before the RSM model is activated, the $\kappa-\varepsilon$ model was applied to prepare the flow field. This also confirmed the inability of $\kappa-\varepsilon$ model to simulate outer bank cell. The velocity fields are plotted by taking their components at $60^{\circ}$ as Fluent uses a Cartesian reference system. The validation results are shown for the outer half section at $60^{\circ}$ since this is where the original measurements were taken.

It can be seen that the distribution of velocity contours is not symmetric and is shifted outwards towards the outer bank of the bend owing to the presence of secondary flows. The value approaches zero near the side walls from the maximum velocity (near the outer bank) due to boundary layer formation. The average velocity of flow is $0.38 \mathrm{~m} / \mathrm{s}$. The model predicts a maximum value of around $0.44 \mathrm{~m} / \mathrm{s}$ whereas the experimental plot shows the same value as $0.56 \mathrm{~m} / \mathrm{s}$. The location of the maximum velocity contour is also of interest and is found well below the free surface. The horizontal position of the maximum velocity contour is found at the intersection of both the circulation cells which agrees well with the experimental data.

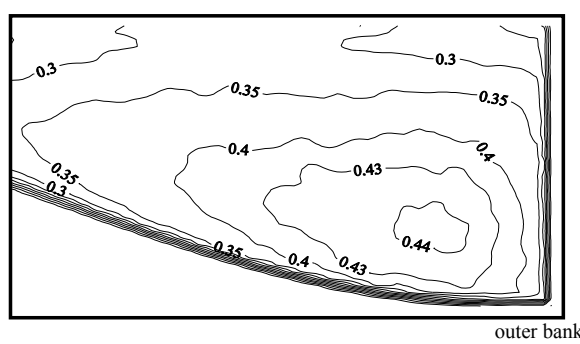

2(a) Simulated

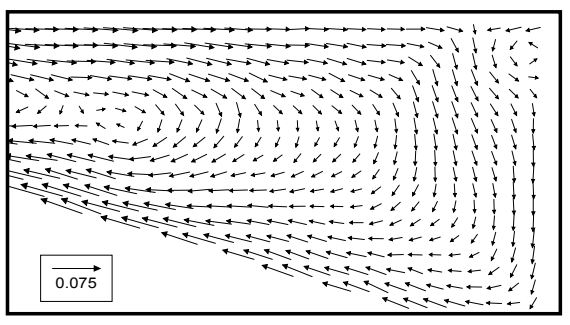

2(c) Simulated

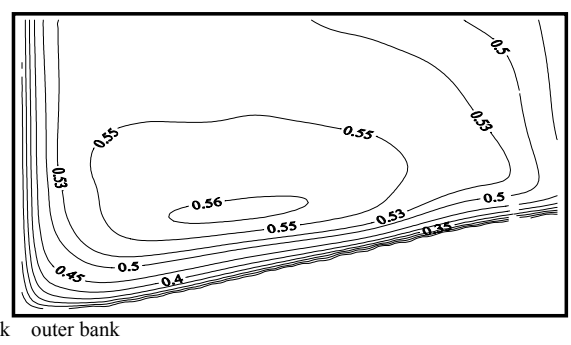

2(b) Measured

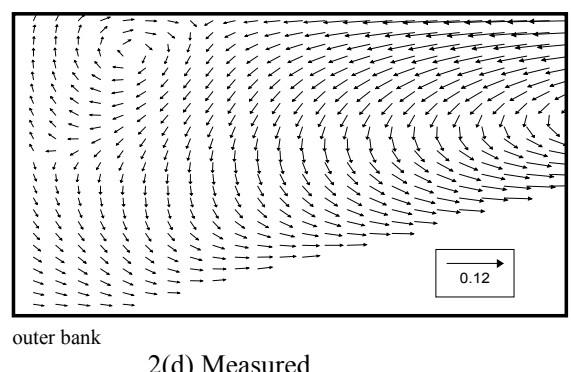

2(d) Measured

Figure 2: $\quad$ Simulated and measured $(\mathrm{a}, \mathrm{b})$ velocity contours and $(\mathrm{c}, \mathrm{d})$ vectors at $60^{\circ}$ section. 
Blanckaert and de Vriend [7] indicated that the resulting decreasing velocities towards the water surface are an important generation mechanism for the outerbank cell. The predicted vector plot (Figure 2c) shows that both the circulation cells are well simulated. In the measured vector plot, the velocity vectors at the bottom are pointing horizontally which must be due to a lack of proper experimentation near the bed, so care should be taken with their interpretation with the measured values. The simulation underestimates the strength of both the circulation cells. It can also be observed that the predicted length of the outer bank cell is less than that of the measured one. This also leads to the conclusion that the underestimation of the strength of the centre region cell leads to the underestimation of the outward increase of the longitudinal velocity contours. Blanckaert and Graf [6] also concluded that the outer bank cell has a protective effect on the outer bank because the outer bank cell keeps the core of the maximum velocity away from the bank.

Figure 3 presents the normalized distribution of the downstream velocity, $V_{s n}$, and the unit discharge, $Q_{s n}$, in the outer half-section. It can be seen that the flow is concentrated over the deeper part of the section and the majority of the discharge flows through the investigated half-section. The depth-averaged values of the downstream velocity remain almost constant throughout the outer halfsection and reduce to zero near the side wall. The value for normalized velocity and discharge is calculated by:

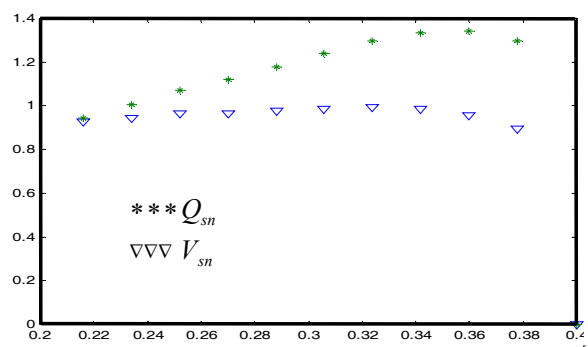

$$
\begin{gathered}
V_{s n}=\frac{1}{h} \int_{z_{b}}^{z_{t}} v_{s} d z \\
Q_{s n}=V_{s n} B h / Q
\end{gathered}
$$

where ' $\mathrm{B}$ ' is width, ' $\mathrm{h}$ ' is local depth of flow, subscript ' $t$ ' and ' $b$ ' denotes free surface and bottom of the channel

Figure 3: Normalized depth-averaged velocity $\left(V_{s n}\right)$, and normalized unit discharge $\left(Q_{s n}\right)$.

\subsection{Mean-flow kinetic energy}

Figure 4 shows the normalized distribution of mean kinetic energy. The kinetic energy per unit mass is defined as:

$$
K=\frac{1}{2}\left(V_{\sigma}^{2}+V_{\eta}^{2}+V_{z}^{2}\right)
$$




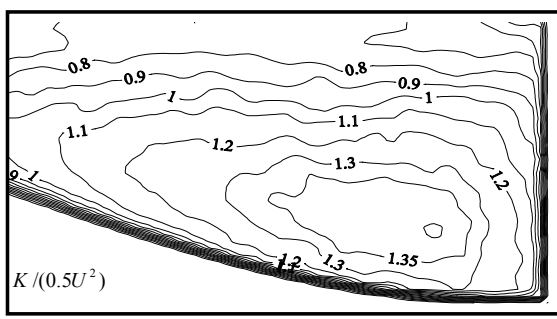

(a) Simulated

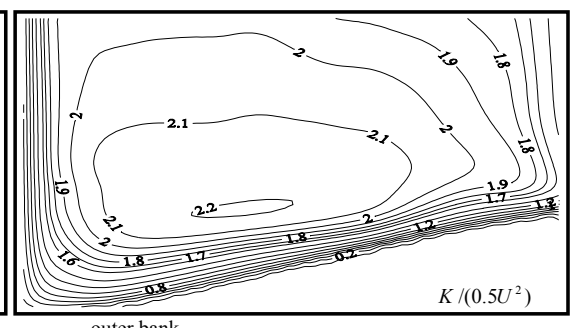

(b) Measured

Figure 4: $\quad$ (a) Simulated and (b) measured mean-flow kinetic energy.

The figure depicts that the simulated results match well with the measured ones. The variation of kinetic energy contours is more or less similar to the longitudinal velocity contours where the respective maximum value is near the outer bank owing to the presence of secondary flows. The computational model predicts the maximum value of around 1.35 whereas the measured plot shows the same value is 2.2. This difference in the magnitude between the two can be ascribed to the under prediction of velocity contours. The comparison between the longitudinal velocity contours and kinetic energy contours shows that the longitudinal component of the velocity dominates the kinetic energy and contains about $98 \%$ of the flow. The K.E. contour values are relatively higher because K.E. varies according to the square of the velocity.

As it can be observed, some discrepancy exists between the experimental and simulated results and more research is required to improve the understanding of the mechanism underlying the formation of outer-bank cell and the under prediction of the velocity magnitudes.

\section{Conclusions}

Secondary flows are significant phenomena of curved open channels which strongly influence the flow behaviour within the natural rivers and estuaries. Besides the classical center-region cell, a counter rotating cell is also observed near the outer bank. The standard $\kappa-\varepsilon$ model has been shown to be unable to simulate this outer-bank cell which confirms the results of previous studies. However, it has been concluded that the RSM model is able to simulate both the circulation cells successfully. The main flow features of the flow field are captured reasonably well although it has not been possible to remove all discrepancies between the model and experimental results. More simulations are being undertaken presently to further improve the predictions with the help of CFD.

\section{Acknowledgements}

The first author would like to thank RPS-MCOS, Ireland for providing funds to carry out research. 


\section{References}

[1] Patel, T. \& Gill, L., Flow characteristics of curved open channel using computational fluid dynamics, ISSEC Annual Symposium, pp. 28, 2005.

[2] Rameshwaran, P. \& Naden, P.S., Modeling turbulent flow in two-stage meandering channels, Proc. ICE, Water Management 157, pp. 159-173, 2004.

[3] Rameshwaran, P. \& Naden, P.S., Three-dimensional modeling of free surface variation in a meandering channel, J. of Hydraulic Research, 42(6), pp. 603-615, 2004.

[4] Ye, J. \& McCorquodale, J.A., Simulation of curved open channel Flows by 3D hydrodynamic model, J. of Hydraulic Engineering, ASCE, 124(7), pp. 687-698, 1998.

[5] Booij, R., Measurements and large eddy simulations of the flows in some curved flumes, J. of Turbulence, 4, pp. 1-17, 2003.

[6] Blanckaert, K., \& Graf, W.H., Mean flow and turbulence in open-channel bend, J. of Hydraulic Engineering, ASCE, 127(10), pp. 835-847, 2001.

[7] Blanckaert, K., \& de Vriend, H.J, Secondary flow in sharp open channel bends, J. of Fluid Mechanics, 498, pp. 353-380, 2004.

[8] Cokljat, D., \& Younis, A., Second-order closure study of open-channel flows, J. of Hydraulic Engineering, ASCE, 121(2), pp. 94-107, 1995.

[9] Leschziner, M.A., \& Rodi, W., Calculation of annular and twin parallel jets using various discretization schemes and turbulence-model variations, J. of Fluid Engineering, ASME Transactions, 103(6), pp. 352-360, 1981.

[10] Nicholas, A.P., Computational fluid dynamics modeling of boundary roughness in gravel-bed rivers: an investigation of the effects of random variability in bed elevation, Earth Surfaces Processes and Landforms, 26, pp. 346-362, 2001.

[11] Fluent Software Manual, version 6.2.16, 2005.

[12] Hirt, C.W., \& Nichols, B.D., Volume of Fluid (VOF) method for the dynamics of free boundaries, J. of Computational Physics, 39, pp. 201225, 1981.

[13] Patankar, S.V., Numerical heat transfer and fluid flow, Hemisphere Publishing, 1980. 